\title{
HilDDD: A high-throughput imaging pipeline for the quantitative detection of DNA damage in primary human immune cells
}

Kelsey Gallant ${ }^{1}$, Arsun Bektas², Mary Kaileh², Ana Lustig², Luigi Ferrucci², Gianluca Pegoraro $^{1}$ and Tom Misteli ${ }^{1}$

${ }^{1}$ National Cancer Institute, NIH, Bethesda, MD, 20892, USA

${ }^{2}$ National Institute on Aging, NIH, Baltimore, MD 21224, USA

Correspondence: mistelit@mail.nih.gov 


\section{Abstract}

DNA damage is a prominent biomarker for numerous diseases, including cancer and aging. Detection of DNA damage routinely relies on traditional microscopy or cytometric methods. However, these techniques are typically of limited throughput and are not ideally suited for large-scale longitudinal and population studies that require analysis of large sample sets. We have developed HiIDDD (High-throughput Immune cell DNA Damage Detection), a robust, semiquantitative and single-cell assay that measures DNA damage by highthroughput imaging using the two major DNA damage markers 53BP1 and $\gamma-\mathrm{H} 2 \mathrm{AX}$. We demonstrate sensitive detection of DNA damage in a wide set of freshly isolated and cryopreserved primary human immune cells, including CD4 ${ }^{+}$ and $\mathrm{CD}^{+} \mathrm{T}$ cells, B cells and monocytes with low inter-assay variability. As proof of principle, we demonstrate parallel batch processing of several immune cell types from multiple donors. We find common patterns of DNA damage in multiple immune cell types of donors of varying ages, suggesting that immune cell properties are specific to individuals. These results establish a novel highthroughput assay for the evaluation of DNA damage in large-scale studies. 


\section{Introduction}

A large number of DNA lesions occur daily in a human cell. DNA damage is caused by exogenous triggers, such as cytotoxic chemicals or irradiation, and by endogenous events such as DNA replication ${ }^{1-3}$. Cells counteract DNA damage through several DNA damage response (DDR) pathways that are activated based on the type of lesion present $^{1,2,4,5}$. Homologous recombination (HR) uses sister chromatids as a template for repair and acts both on single- and double strand breaks (DSBs) and is limited to the Sand G2-phase of the cell cycle. The majority of DSBs, however, are eliminated by nonhomologous end joining (NHEJ), in which DSBs are repaired by ligation of the gap without use of a template, often leading to short deletions or insertions ${ }^{6}$.

NHEJ requires a complex protein machinery to execute the repair process ${ }^{7,8}$. In human cells the core histone variant $\mathrm{H} 2 \mathrm{AX}$ is phosphorylated at Ser139 $(\gamma-\mathrm{H} 2 \mathrm{AX})$ in response to DNA damage ${ }^{9}$. Once established, $\gamma-\mathrm{H} 2 \mathrm{AX}$ acts as a binding site for various repair factors that ultimately execute the repair reaction ${ }^{7,8}$. One prominent NHEJ repair factor is the tumor suppressor p53-binding protein 1 (53BP1), which colocalizes with $\square-\mathrm{H} 2 \mathrm{AX}$ and promotes NHEJ-mediated repair ${ }^{10,11}$. The accumulation of $\square-\mathrm{H} 2 \mathrm{AX}$ and 53BP1 is detected as distinct foci in the nucleus of cells that contain DNA damage and can be visualized with high reliability by indirect immunofluorescence using specific antibodies $^{7,10-12}$. Because of their prominent involvement in NHEJ and robust detection, both 53BP1 and $\square-\mathrm{H} 2 \mathrm{AX}$ have been widely used as biomarkers of DSBs and of DDR activity $^{12-15}$.

DNA damage is a physiologically relevant biomarker for various diseases and pathological conditions, including cancer and inflammation, and it is routinely used in the assessment of immunotherapy effects, drug action, and radiation exposure ${ }^{16-18}$. Determination of DNA damage is of particular interest in monitoring human aging since genome instability and increased DNA damage have been proposed to contribute to the aging process ${ }^{19-21}$. In support of this view, many premature aging disorders, such as Werner Syndrome, Cockayne Syndrome or Hutchinson Gilford Progeria Syndrome, are characterized by increased DNA damage ${ }^{22,23}$. Moreover, an elevated mutational load in DNA repair-associated genes has been linked to numerous aging-related cancers ${ }^{17,24,25}$. 
However, the extent to which DNA repair capacity contributes to the normal aging process remains unclear.

Standard methods to quantitatively measure DNA damage accumulation and DNA repair capacity in human cells are well established and include use of alkaline comet assays, indirect fluorescence microscopy using antibodies and flow cytometry ${ }^{5,26-31}$. However, these assays are often limited in that they either measure bulk DNA damage, as in the case of comet assays or are of low throughput, such as microscopy and cytometry methods. These limitations make these assays unsuitable to probe large numbers of primary human samples that would be needed for comprehensive longitudinal or epidemiological studies, and to assess the role of DNA damage in many physiological settings.

We have developed here a versatile assay for high-throughput detection of DNA damage in multiple primary immune cell types. The assay is based on the detection of the two prominent DNA damage markers $\square-\mathrm{H} 2 \mathrm{AX}$ and 53BP1 using a high-throughput imaging platform. Peripheral blood mononuclear cells (PBMCs) are a suitable tissue source for large-scale studies since they can be easily collected, and since they serve as a sensitive and global indicator of an individual's physiological status. However, immune cells and PBMCs tend to be a challenge in microscopy-based approaches due to their fragility and poor adherence to substrates used in most imaging methods ${ }^{32,33}$. We have overcome these limitations by optimizing an assay, HilDDD (High-throughput Immune cell DNA Damage Detection), which uses immunofluorescence (IF) and highthroughput imaging $(\mathrm{HTI})$, combining automated liquid handling, microscopy, and image analysis (Fig. 1a). This approach allows imaging and quantitative analysis of up to tens of thousands of individual cells in a large numbers of samples in a single experiment $^{34,35}$. In HilDDD, we have optimized cell seeding, fixation, permeabilization, and immunostaining steps to create a robust HTI IF protocol for detection of DNA damage on immobilized T cells, B cells, and monocytes in 384-well microplates. We demonstrate that this approach is a sensitive and reliable assay for the measurement of DNA damage in primary human immune cells. 


\section{Results}

\section{Detection of DNA damage in immune cell lines in 384-well format}

We sought to establish a high-throughput imaging pipeline as a rapid and robust method for the detection and measurement of DNA damage and repair capacity in immune cells. The HilDDD pipeline consists of plating of immune cells in 384-well plates, detection of $\square-\mathrm{H} 2 \mathrm{AX}$ and 53BP1 by IF, automated imaging, image analysis, and statistical analysis (Fig. 1a). For HilDDD development, a set of semi-automated steps for sample preparation, indirect immunofluorescence (IF) staining, and washing methods were optimized to maximize the adherence of a range of immune cell types and to minimize technical variability between replicates (Fig. 1; see Materials and Methods). Primary antibodies against two prominent biomarkers of DNA damage and repair, 53BP1 and $\square-\mathrm{H} 2 \mathrm{AX}$, were used based on their previously demonstrated efficiency in IF staining protocols for multiple cell types. The assay was designed to be amenable to a wide range of high-content data acquisition and analyses parameters for cellular features detected (e.g., foci number, foci intensity, nuclear intensity, etc.).

Most immune cells grow in suspension and are notoriously difficult to immobilize on a glass or plastic surface, which is generally necessary for imaging. To optimize the immobilization of immune cells on a substrate for $\mathrm{HTI}$ with automated liquid handling, we explored use of various surface coatings including large surface glycoproteins (e.g. CD45 and CD43) or activated polyethylene glycol (e.g. SUNBRIGHT ${ }^{\circledR}$ ) previously shown to promote immune cell attachment ${ }^{36}$, but found only a modest increase in adhesion compared to routinely used poly-D-lysine coating alone (Fig. 1b). We thus first optimized cell seeding density and evaluated adherence of Jurkat, BJAB and THP-1 cell lines, representing $T$ cells, B cells and monocytes, respectively, on poly-D-lysine coated 384-well plates (see Materials and Methods). To determine optimal cell seeding density, cells were plated in 384-well plates in a range from $2 \times 10^{4}$ cells/well to $1 \times 10^{5}$ cells/well in a total volume of $40 \mu \mathrm{l}$ per well (Fig. 1c). Cells were fixed and plates were centrifuged briefly (400x g, $4 \mathrm{~min}, \mathrm{RT}$ ) and post-fixed in 4\% paraformaldehyde for $20 \mathrm{~min}$ at RT. Importantly, single fixation before or after centrifugation of cells and use of a robotic dispenser resulted in significantly lower cell yields (Fig. 1b). Using manual dispensing 
onto poly-D-lysine coated wells, seeding density was evaluated based on the number of recovered cells detected by DAPI and variability between four technical replicates for each density after staining for $\square-\mathrm{H} 2 \mathrm{AX}$ or 53BP1. The optimal seeding density was determined as $\sim 0.6-1.0 \times 10^{5}$ cells/well for Jurkat and $\sim 8 \times 10^{4}$ cells/well for BJAB and THP-1 cells (Fig. 1c).

Detection of DNA damage by HTI in immune cell lines

In order to determine whether an increase in DNA damage can be detected by $\mathrm{HTI}$, Jurkat, THP-1, and BJAB cells were treated with $30 \mu \mathrm{M}$ of etoposide (ETP) for $1.5 \mathrm{hrs}$ to induce dsDNA breaks (DSB) and cells were subjected to HTI immunostaining for 53BP1 and $\square-\mathrm{H} 2 \mathrm{AX}$ (Fig. 2a). For each condition, three technical replicate wells were imaged in nine randomly selected fields of view (FOV) per well, corresponding to approximately $1-5 \times 10^{3}$ cells per condition, and single-cell signal intensity for 53BP1 and $\square-\mathrm{H} 2 \mathrm{AX}$ was measured using automated image analysis (see Materials and Methods). Typically, 2,000-5,000 cells were analyzed per sample. Distinct fluorescence intensity parameters were used for the two markers based on their different changes in nuclear fluorescence distribution in response to DNA damage in the primary immune cells used here. For 53BP1, which is present in the cell nucleus prior to DNA damage but accumulates in distinct foci upon DNA damage, integrated spot intensity was measured, whereas for $\square$ $\mathrm{H} 2 \mathrm{AX}$, which is present at low levels in undamaged cells and rapidly increases upon DNA damage due to phosphorylation at $S 139^{\text {ref.9 }}$ total mean nuclear intensity was measured $^{10,11}$. As expected, upon ETP treatment 53BP1 and $\square-\mathrm{H} 2 A X$ rapidly formed nuclear foci at sites of DNA damage (Fig. 2a) and we observed an increase in both 53BP1 and $\square-\mathrm{H} 2 \mathrm{AX}$ pan nuclear staining, defined as the detected fluorescence of $\square$ H2AX within segmented nuclei (Fig. 2a).

In Jurkat cells, integrated 53BP1 spot intensity per cell increased significantly upon DNA damage by ETP (median: $1.06 \pm 0.93$ e6 A.U.; standard deviation) compared to vehicle control (median: 4.67 SD: \pm 5.85 e5 A.U.) (Wilcoxon test p-value < 2.2 e-16) (Fig. 2b). Importantly, using single cell analysis we observed an increase in the number of cells that were 53BP1 positive when treated with ETP compared to the vehicle treatment 
(69.0\% versus 33.4\%; $X^{2}=518.49, p<2.2$ e-16; Supplementary Fig. 1) when applying a threshold of one standard deviation (SD) from the mean of the negative control. Likewise, ETP-treated Jurkat cells (median: $432 \pm 459$ A.U) showed a significant increase in $\square-\mathrm{H} 2 \mathrm{AX}$ mean nuclear intensity when compared to the vehicle control (median: $199 \pm 151$ A.U) (Fig. 2c; Wilcoxon test $p$-value $p<2.2$ e-16). At the single cell level, $\square-\mathrm{H} 2 \mathrm{AX}$ was detected in $42.3 \%$ of ETP-treated cells compared to $12.4 \%$ in untreated controls cells using as a threshold one standard deviation from the mean $\square$ $\mathrm{H} 2 \mathrm{AX}$ nuclear intensity of the negative control (Fig. 2c; Supplementary Fig. $1 ; X^{2}=$ 499.09, $p<2.2$ e-16; DMSO: $\mathrm{n}=2645$ ETP: $\mathrm{n}=1654$ ).

Similar results were found for BJAB and THP-1 cells (Fig. 2b). For BJAB cells, integrated 53BP1 spot intensity per cell increased about 5-fold from $3.21 \pm 0.71$ e4 A.U. in controls to $1.78 \pm 1.57$ e5 A.U. in ETP-treated cells (Fig. 2b, Wilcoxon test p-value < $2.2 \mathrm{e}-16)$. In addition, $41.8 \%$ of cells were 53BP1-positive when compared to the vehicle treatment $(5.4 \%)\left(X^{2}=2057.2, p<2.2 \mathrm{e}-16\right)$. $\square-\mathrm{H} 2 \mathrm{AX}$ mean nuclear intensity increased from $20.1 \pm 5.79$ A.U. in controls to $72.8 \pm 28.1$ A.U. in ETP-treated samples (Fig. 2c, Supplementary Fig. 1; middle, Wilcoxon test $p$-value $<2.2$ e-16). At the single cell level, $\square-\mathrm{H} 2 \mathrm{AX}$ was detected in $75.9 \%$ of ETP-treated cells compared to $5.7 \%$ in untreated controls cells (Fig. 2c, Supplementary Fig. $1 ; X^{2}=5855.1, p<2.2$ e-16; DMSO: $n=$ 5497 ETP: $n=6193)$.

For THP-1 monocytes, we found a significant increase in integrated 53BP1 spot intensity per cell in response to ETP-induced DNA lesion (median: $6.25 \pm 6.07$ e5 A.U.) as compared to vehicle control (median: $3.37 \pm 2.95$ e5 A.U.) (Wilcoxon test p-value < $2.2 \mathrm{e}-16$ ) (Fig. 2b). Similarly, 32.4\% of cells were 53BP1 positive when compared to the vehicle treatment $(13.6 \%)\left(X^{2}=177.36, p<2.2 \mathrm{e}-16\right)$. As expected, there was also an increase in $\square-\mathrm{H} 2 \mathrm{AX}$ mean nuclear intensity (Fig. 2c, Supplementary Fig. 1; Wilcoxon test $p$-value $<2.2$ e-16) when comparing ETP-treated cells (median: $317 \pm 250$ A.U.) to vehicle control (median: $196 \pm 117$ A.U.). At the single cell level, $\square-\mathrm{H} 2 \mathrm{AX}$ was detected in $9.46 \%$ of ETP-treated cells compared to $5.65 \%$ in untreated controls cells using as a threshold of one SD from the mean of $\square-\mathrm{H} 2 \mathrm{AX}$ mean nuclear intensity in the negative 
control (Fig. 2c, Supplementary Fig. 1; $X^{2}=3.71, p=0.054 ;$ DMSO: $n=1591$ DMSO: $n=2348)$.

To relate the behavior of the two DNA damage markers to each other and to ask whether both markers simultaneously increased in individual cells in response to ETP, or if they represent cell-autonomous events, integrated 53BP1 spot intensity per cell and $\square-\mathrm{H} 2 \mathrm{AX}$ mean nuclear intensity per cell were plotted as two-dimensional kernel density estimates (Fig. 2d). ETP treatment shifted the density distributions to higher values of both parameters in all cell types compared to untreated conditions, suggesting concomitant responses in most cells in the population (Fig. 2d).

Detection and measurement of 53BP1 and $\square-H 2 A X$ in primary immune cells using $H T I$

To assess whether HilDDD can detect and quantitatively measure DNA damage in primary immune cells, we applied the pipeline to freshly isolated and frozen CD4 ${ }^{+} T$ cells, CD8 ${ }^{+}$T cells, B cells and monocytes. Similar to cultured cell lines, and following ETP treatment, isolated immune cells were immediately fixed in 4\% PFA and spun onto 384-well plates and processed for 53BP1 and $\square-\mathrm{H} 2 \mathrm{AX}$ staining (see Materials and Methods). The staining patterns of 53BP1 and $\square-\mathrm{H} 2 \mathrm{AX}$ in the presence and absence of ETP-induced DNA damage were similar to those seen in immune cell lines (Fig. 3a). Freshly isolated and frozen cell isolates yielded identical results.

Quantitation of integrated 53BP1 spot intensity per cell for $C D 4^{+} T$ cells showed an increase in response to ETP treatment (median: $8.71 \pm 6.23$ e5 A.U.) as compared to untreated cells (median: $5.24 \pm 2.98$ e4 A.U.) (Wilcoxon test p-value $<2.2$ e-16) (Fig. $3 b)$. In untreated cells, only $35.7 \%$ of cells were 53BP1 positive as compared to $66.8 \%$ of ETP-treated cells $\left(X^{2}=306.43, p<2.2\right.$ e-16). In ETP-treated cells, $\square-\mathrm{H} 2 \mathrm{AX}$ mean nuclear intensity (median: $993 \pm 652$ A.U.) increased compared to untreated cells (median: $527 \pm 112$ A.U.) (Wilcoxon test p-value < 2.2 e-16) (Fig. 3c). Similarly, 47.3\% of ETP-treated cells that had a nuclear intensity classified as positive- $\square-\mathrm{H} 2 \mathrm{AX}$ whereas 
only $33.3 \%$ showed H2AX positivity in untreated cells (Fig. 3c; $X^{2}=446.79$, p-value $<2.2 \mathrm{e}-16)$.

We similarly detected DNA damage in all other primary immune cells. In CD8+T cells integrated 53BP1 spot intensity per cell increased from $4.58 \pm 2.27$ e4 A.U. to $8.36 \pm$ 5.93 e4 A.U. in ETP-treated cells (Fig. 3b ; Wilcoxon test, $p<2.2$ e-16). We also found $69.3 \%$ of the cells in the ETP-treated condition were positive for 53BP1 as compared to $23.7 \%$ in the untreated condition $\left(X^{2}=875.77, p<2.2\right.$ e-16). Furthermore, there was a significant increase in $\square-\mathrm{H} 2 \mathrm{AX}$ mean nuclear intensity in ETP-treated cells compared to untreated cells (median: $1266 \pm 641$ A.U. vs. $623 \pm 244$ A.U.; Wilcoxon test p-value $p<$ 2.2 e-16) (Fig. 3c). Similarly, 13.5\% of ETP-treated cells that had a nuclear intensity classified as positive- $\square-\mathrm{H} 2 \mathrm{AX}$ using a threshold of one SD above the mean $\square-\mathrm{H} 2 \mathrm{AX}$ nuclear intensity of the negative control, whereas only $0.2 \%$ of untreated cells were positive for H2AX (Fig. 3c; $X^{2}=357.51$, p-value <2.2 e-16).

B cells showed a significant increase in integrated 53BP1 spot intensity per cell upon ETP-treatment (median: $1.33 \pm 0.85$ e5 A.U.) when comparing to untreated cells (median: $4.76 \pm 1.84$ e4 A.U.; Wilcoxon test, $p<2.2$ e-16) (Fig. 3b). Moreover, 51.9\% of cells were 53BP1 foci positive in ETP treated cells compared to $6.25 \%$ in untreated cells $\left(X^{2}=1041.5, \mathrm{p}<2.2 \mathrm{e}-16\right)$. We also observed a significant increase in $\square$-H2AX nuclear intensity of ETP-treated B cells (median: $1368 \pm 790$ A.U.) when compared to untreated B cells (median: $801 \pm 201$ A.U., Wilcoxon test $p<2.2$ e-16) (Fig. 3c). Similarly, 61.5\% of ETP-treated cells that had a nuclear intensity classified as positive- $\square-\mathrm{H} 2 \mathrm{AX}$ compared to only $10.2 \%$ of untreated control cells (Fig. 3c, $X^{2}=584.03$, p-value $<2.2$ e16).

For monocytes, we found a significant increase in integrated 53BP1 spot intensity per cell in response to ETP-induced DNA lesion (median: $5.00 \pm 2.68$ e4 A.U.) as compared to untreated control (median: $3.72 \pm 0.99$ e4 A.U.; Wilcoxon test p-value $p=3.7$ e-3) (Fig. 3b). The number of cells positive for 53BP1 was not statistically different between control and ETP treated cells $\left(6.0 \%\right.$ vs $\left.9.4 \% ; X^{2}=3.4476, p=0.06\right)$. There was also a significant increase in $\gamma-\mathrm{H} 2 \mathrm{AX}$ nuclear intensity when we compared ETP-treated cells 
(median: $3608 \pm 1361$ A.U.) to untreated cells (median: $1484 \pm 382$ A.U.; Wilcoxon test $\mathrm{p}<2.2$ e-16) (Fig. 3c). Similarly, 89.9\% ETP-treated cells that had a nuclear intensity classified as positive- $\square-\mathrm{H} 2 \mathrm{AX}$ using a threshold of one SD above the mean $\square-\mathrm{H} 2 \mathrm{AX}$ nuclear intensity of the negative control, compared to only $10 \%$ of untreated cells (Fig. $3 c, X^{2}=607.38 ;$ p-value $<2.2$ e-16).

Two-dimensional kernel density mapping confirmed the observed responses in all cell types (Fig. 3d) and the diagonal shift between control and ETP treated samples indicated that both DDR markers were simultaneously induced in individual cells. Taken together, these results demonstrate that a wide range of immune cells are amenable to quantitative interrogation of their DNA damage status in a high-throughput imaging format and that the extent of DNA damage can be sensitively measured in this assay.

\section{Inter-assay variability in $C D 4^{+} T$ cells}

In order to assess the robustness of HiIDDD, and to test whether this pipeline can be used to compare different biological samples in multiple batches, we determined the technical variability of our HTI pipeline between samples obtained from a single donor. To do so, we used three samples of frozen $\mathrm{CD}^{+} \mathrm{T}$ cells from a single donor that were independently thawed, IF stained, imaged, and analyzed in different experimental batches (i.e., on different days). Cells were either left untreated, treated with DMSO as a vehicle, or treated with $30 \mu \mathrm{M}$ ETP for $1.5 \mathrm{hrs}$. The 53BP1 integrated spot intensity per cell (Fig. 4a) and $\square-\mathrm{H} 2 \mathrm{AX}$ mean nuclear intensity per cell (Fig. 4b) were quantitated for each replicate and the variability of the biological replicates for each treatment group was analyzed via ANOVA. As expected ETP treatment significantly increased 53BP1 spot intensity $($ mean $=1.36 \pm 1.04$ e5) compared to DMSO vehicle control $($ mean $=2.17$ \pm 4.24 e4) and untreated cells (mean $=0.9171 \pm 2.46$ e4) (Fig. $4 a, p=1.9 e-4)$. We similarly observed a modest increase in $\square-\mathrm{H} 2 \mathrm{AX}$ nuclear intensity when ETP-treated cells (mean $=2248 \pm 2304$ ) were compared to DMSO controls (mean = $811 \pm 2174$ ) or to untreated cells (mean $=770 \pm 2678$ ) (Fig. $4 b, p=0.011)$. No statistically significant differences were observed between individual replicates for 53BP1 spot intensity $(p=$ 
$0.99)$ nor for $\square-H 2 A X$ nuclear intensity $(p=0.93)$. These results demonstrate that the HTI IF assay is robust and shows negligible batch to batch, or technical, variability.

\section{Batch processing of primary human immune cells}

The intent of developing a high-throughput imaging assay for detection of DNA damage is its use in analyzing large sample numbers such as from archived materials that are part of longitudinal studies. This application would require batch processing of samples. To demonstrate scalability of HiIDDD, three primary immune cell types from 10 different donors were processed simultaneously (Supplementary Fig. 2). Cells, either untreated or treated with $30 \mu \mathrm{M}$ ETP ex-vivo for $1.5 \mathrm{hrs}$, were processed in parallel and seeded in triplicate wells on a 384-well plate for imaging (see Materials and Methods). Elevated DNA damage, as indicated by an increase in 53BP1 and $\square-\mathrm{H} 2 \mathrm{AX}$ staining intensity, was detected in all samples to similar extents as in single processed samples (Fig. 5a, b), demonstrating scalability of the assay.

Within each of the ten samples from different donors, the integrated 53BP1 spot intensity varied typically over a $\sim 5$-fold range for all cell types. Similarly, $\square-\mathrm{H} 2 \mathrm{AX}$ mean nuclear intensity values in untreated cells were consistently distributed over an $~ 8-10$ fold range across samples from different donors, in line with values observed in samples from individuals (Fig. 5a-c). Among the samples from the ten donors, the integrated 53BP1 median spot intensity showed limited variation of $\sim 2$-fold in CD4 ${ }^{+} \mathrm{T}$ cells, $\sim 2$-fold in $\mathrm{CD8}^{+} \mathrm{T}$ cells, and $\sim 1$.5-fold in monocytes (Fig. 5A-C). Similarly, for $\square$ H2AX median values varied between samples by $\sim 1.5$-fold in $C D 4^{+} \mathrm{T}$ cells, $\sim 1.5$-fold in CD8 ${ }^{+} \mathrm{T}$ cells, and $\sim 2.5$-fold in untreated monocytes (Fig. 5a-c).

We next challenged all ten samples by ETP-treatment to probe for potential differences in the extent to which the DDR is induced in individual donors. Increased 53BP1 and $\square$ H2AX signals were detected in all samples and cell types (Fig. 5a-C). The distribution for both 53BP1 and $\square-\mathrm{H} 2 \mathrm{AX}$ broadened somewhat within each sample but largely stayed within a $\sim 6$-fold range for 53BP1 and $\sim 10$ fold for $\square-\mathrm{H} 2 \mathrm{AX}$ (Fig. 5a-c). Comparing amongst the ten donor samples after ETP-treatment similar variation of $\sim 2$-fold was 
observed for both 53BP1 median spot intensity and $\square-\mathrm{H} 2 \mathrm{AX}$ median values (Fig. 5a-c). The observed diagonal shift of 53BP1 and $\square-\mathrm{H} 2 \mathrm{AX}$ signal between untreated and treated cells in the dual parameter analysis also confirmed a concomitant increase in both damage markers in individual cells of all samples upon ETP treatment (Fig. 5a-c). No clear correlation between age or sex of the donor and baseline DNA damage was observed amongst the 10 samples derived from donors aged 30 to 85 (Fig. 5a-c). Interestingly, the extent of the response to ETP-treatment, however, appeared to differ between samples, with donors 1,2, 5, and 10 showing less pronounced increases in DNA damage markers upon ETP treatment compared to the other donors (Fig. 5a-c). Importantly, the differences in response were seen concomitantly in all cell types of each individual donor. The coordinated response in multiple immune cells from the same donor points to inherent differences in the DNA damage response between individuals. 


\section{Discussion}

We have here developed HilDDD, an imaging-based method for the detection of DNA damage in a wide variety of primary immune cells in a high-throughput format. HiIDDD is based on an optimized high-throughput imaging pipeline using a combination of both automated and manual techniques to maximize cell recovery following IF staining. We show that this assay is sensitive, robust and can be performed in a batch-processing format to assess both baseline DNA damage levels as well as DDR induction in primary immune cells.

HilDDD relies on the detection of nuclear signals of the two well established DNA damage markers 53BP1 and $\square-H 2 A X^{7,8}$. The method described here extends current approaches, such as cytometric analysis and traditional IF of H2AX phosphorylation status, that have previously been used to assess DNA damage ${ }^{5,26-29}$, in that it generates single cell data and does so in a high-throughput format suitable for largescale studies. The sensitivity of 53BP1 and $\square-\mathrm{H} 2 \mathrm{AX}$ detection was comparable to standard low-throughput microscopy assays and induction of DNA damage by ETP resulted in a robust increase in signal for both 53BP1 and $\square-\mathrm{H} 2 \mathrm{AX}$. The two measured signals are qualitatively distinct, and complementary, in that $\square-\mathrm{H} 2 \mathrm{AX}$ measures the denovo formation of the epitope through phosphorylation of Ser139 on H2AX, whereas the 53BP1 signal measures the accumulation of the protein at sites of DNA damage. To capture these different properties of the two markers, mean nuclear intensity was measured for $\square-\mathrm{H} 2 \mathrm{AX}$, but for 53BP1 the intensity of the signal in nuclear foci was detected. The bi-modal distribution of the 53BP1 signal reflects the presence of foci, albeit typically smaller in size, in undamaged cells. Reassuringly, the two DNA damage markers yielded corresponding responses with most cells exhibiting an increasing in both markers upon DNA damage. Importantly for an assay designed for high-throughput applications, we show low variability among cells from the same individual processed in different experimental batches, and high concordance in both basal and induced levels of the DDR in different cell types from the same individual. These observations indicate that HiIDDD is suitable for processing and analysis of large cohorts of samples in multiple separate experimental batches. Moreover, we find that our assay yields 
comparable results regardless of the use of fresh or cryopreserved primary immune cells, making it potentially suitable for interrogation of collections of archived materials.

While HiIDDD has been developed and optimize for the detection of DNA damage, the conditions used can similarly be applied to the detection of any cellular marker of choice. An important step in the detection pipeline is the efficient immobilization of immune cells on the imaging substrate, which has been a notoriously challenging aspect of imaging of immune cells. We describe here optimized methods to ensure sustained adherence of immune cells on the imaging substrate throughout the detection protocol by the combined application of a pre- and post-centrifugation fixation step to maximize the number of immobilized cells and the use of sponge evacuation for aspiration of fluids during all incubation steps rather than vacuum-mediated aspirations (see Methods). Using these steps, we typically find substantial retention of cells through the IF staining protocol, yielding enough cells for high-throughput imaging and collection of sufficiently large single-cell datasets for statistical analysis.

HilDDD enables batch processing of relatively large sample sets. As proof of principle, we analyzed baseline DNA damage and DDR response capacity for 30 samples from ten donors using parallel processing. The total parallel processing of all samples timedivided into sample preparation, IF, and imaging, requires about 3 days with the most time-intensive steps being sample preparation and the manual fixation of seeded cells. In our experimental setup (see Materials and Methods) the imaging time per sample using three technical replicates is $\sim 3 \mathrm{~min}$. Processing time could be reduced by use of more extensive robotics for liquid handling during fixation and DNA damage induction or the use of X-ray or gamma-irradiation of entire 384-well plates without the need for drug treatment and washing. The versatility and capacity for high-throughput make the HilDDD imaging pipeline useful for basic research applications that involve larger sample sets, for longitudinal or natural history studies, and also for clinical applications such as the assessment of immunotherapy responses.

One limitation in our current work is the inherent variability of 53BP1 and $\square-\mathrm{H} 2 \mathrm{AX}$ foci morphology, and of the signal intensity amongst different immune cell types (Fig. 2, 3, 5). A potential strategy to gain more precision is to increase the utility of our detection 
method is the use of additional biomarkers. The two current markers could be combined with other DNA damage markers such as components of the MRE11 complex ${ }^{37}$ or downstream effectors such as CHEK1 or CHEK2 ${ }^{10,37-39}$. Alternatively, non-DNA damage related indicators such as epigenetic markers, could be included in a multiplexed assay. By using multi-dimensional analysis, it should be possible to more accurately cluster single-cell data to reflect DNA damage status.

In summary, we have developed a high-throughput imaging pipeline to detect and quantitatively measure DNA damage in primary human immune cells. Our assay can detect both baseline levels of DNA damage between samples in addition to DNA damage response capacity. The assay is scalable and sensitive to differences in DNA damage response between individuals and represents a robust and easy strategy to measure DNA damage response in immune cells in large sample sets. 


\section{Materials and Methods}

\section{Cell culture}

The Jurkat (Clone E6-1, TIB-152) and THP-1 (TIB-202) cell lines were purchased from ATCC. BJAB cells were a kind gift of Ranjan Sen Lab (National Institute of Aging/NIH). All cell lines were cultured in high glucose Dulbecco's modified eagle's medium (DMEM, ATCC, cat. number 30-2002), 10\% fetal bovine serum (Gibco, cat. number A3410), and $1 \%$ penicillin/streptomycin (Gibco, cat. number 15140122). All cell lines were grown at $37^{\circ} \mathrm{C}$ and $5 \% \mathrm{CO}_{2}$. Cells were maintained according to vendor's recommendations and kept at low passage numbers ( $\leq 6$ passages).

Primary cells processing

Study samples were derived from Genetic and Epigenetic Signatures of Translational Aging Laboratory Testing (GESTALT) participants $(n=5)$ and normal donors $(n=5)$ ages 20-85 years. Participants enrolled in GESTALT (NIA protocol\# 15-AG-0063) are volunteers who at the time of study enrollment were "healthy" based on eligibility criteria $^{40}$. Normal donors are volunteers who donated apheresis material through the Cytapheresis of Volunteer Donors protocol (NIA protocol\# 03-AG-N316). All individuals were consented for their donations and protocols have been reviewed by the NIH institutional review board.

PBMCs were isolated from apheresis packs using Ficoll-Paque Plus (GE Healthcare, Piscataway, NJ, USA cat. number 17-1440-03) density gradient centrifugation. CD4 ${ }^{+} \mathrm{T}$ cells, $\mathrm{CD}^{+} \mathrm{T}$ cells, B cells, and monocytes were separated from freshly isolated PBMCs using immunomagnetic negative selection cell isolation kits (EasySep Human Cell Enrichment Kits; $\mathrm{CD}^{+}{ }^{+} \mathrm{T}$ cells: cat. number 19052; $\mathrm{CD}^{+} \mathrm{T}$ cells: cat. number 19053; B cells: cat. number 19054 and monocytes, without CD16 depletion: cat\# 19058; StemCell Technologies, Vancouver, BC, Canada) using a fully automated cell separator (RoboSep, StemCell Technologies) according to the manufacturer's protocol.

$\mathrm{CD}^{+} \mathrm{T}$ cells, $\mathrm{CD} 8^{+} \mathrm{T}$ cells, $\mathrm{B}$ cells, and monocytes were cryopreserved in freezing medium (FBS from Life Technologies, cat. number 10437028 and 15\% DMSO from Sigma Aldrich, cat. number D2650) in a freezing container overnight at $-80^{\circ} \mathrm{C}$ and 
transferred to below $-140^{\circ} \mathrm{C}$ liquid nitrogen freezer until use. Cryopreserved cells were thawed using RPMI 1640 containing 10\% fetal calf serum.

\section{Etoposide treatment}

Cell lines and isolated primary human immune cells were treated with etoposide (ETP, Sigma-Aldrich, cat. number E1383) at the indicated concentrations from a $50 \mathrm{mM}$ stock that was prepared from a lyophilized powder reconstituted in DMSO (Sigma-Aldrich, cat. number 20-139). For treatment, cells were seeded at $1.25 \times 10^{6}$ cells $/ \mathrm{ml}$ in 6 -well flat bottom cell culture plates (Corning, cat. number 07-200-83). The incubation time with etoposide or DMSO for all experimental conditions was $1.5 \mathrm{hrs}$ at $37^{\circ} \mathrm{C}$ and $5 \% \mathrm{CO}_{2}$.

Semi-automated sample preparation and immunofluorescence (IF) staining in 384-well plates

For the DNA damage detection assay, cells were washed once with D-PBS (R\&D Systems, cat. number 30250) and fixed with 4\% paraformaldehyde (PFA, Electron Microscopy Sciences, cat. number 50-259-96) in D-PBS for $20 \mathrm{~min}$ at RT. Cells were washed twice with D-PBS and then resuspended in D-PBS before being seeded on 384-well plates with poly-D-lysine coating (PerkinElmer CellCarrier Ultra 384, cat. number 6057500). Plates were pre-warmed at $37^{\circ} \mathrm{C}$ for $20 \mathrm{~min}$ prior to cell seeding. Cells were routinely stored for up to $72 \mathrm{hrs}$ at $4^{\circ} \mathrm{C}$ prior to seeding on plates. Following a centrifugation step (400 g, $5 \mathrm{~min}$, at RT), cells were crosslinked to plates using 4\% PFA for 20 min before washing with D-PBS. All washes of 384-well plates were completed using an automated BlueWasher liquid handler (BlueCatBio) with settings optimized for low-adhering cells. To wash cells, 40-60 $\mathrm{l} /$ well of D-PBS was dispensed at a pressure level of 1 with auto-prime and staccato dispense cadence enabled. Dispense position offset was adjusted to -0.5 . Liquid was gently aspirated by sponge evacuation by placing 384-well plates over a standard utility sponge overlayed with a stainless-steel mesh screen. Pressure was lightly applied to the back of the plate to promote evacuation of wash liquid. This process was repeated 2-3 times. Cells were permeabilized with $0.5 \%$ Triton X-100 (Thermo Scientific, cat. number 85111) diluted in D-PBS for no more than 8 min before washing with D-PBS. Primary and secondary antibodies were suspended in antibody buffer containing $1 \%$ bovine serum albumin 
(BSA, Sigma Aldrich, cat. Number A9418) and $0.1 \%$ Triton X-100 in D-PBS. Primary antibodies were incubated overnight $(8-14 \mathrm{hrs})$ at $4^{\circ} \mathrm{C}$, and secondary antibodies were incubated for $1 \mathrm{hr}$ at RT. Cells were washed twice in D-PBS after each antibody incubation period.

The following antibodies were used: Primary rabbit anti-53BP1 (1:1200, Novus Biological, cat. number NB100-304) and primary mouse monoclonal anti- $\square-\mathrm{H} 2 \mathrm{AX}$ (1:1200, Sigma-Aldrich, cat. number 05-636). 4',6-diamidino-2- phenylindole (DAPI) $(10 \mu \mathrm{g} / \mathrm{ml}$, Biotium, cat. number 40043) was used for nuclear staining. Labelled secondary antibodies were goat anti-mouse AlexaFluor488 (Invitrogen, cat. number A28175) or donkey anti-rabbit AlexaFluor647 (Invitrogen, cat. number A-31573). Both secondary antibodies were used at a concentration of 1:1000.

High throughput image acquisition and analysis

Cells were imaged using a CV7000 (Yokogawa) high throughput spinning disk confocal microscope. Images were acquired using a 60X water-immersion lens (NA 1.2) and 2 sCMOS cameras ( $2560 \times 2160$ px, physical pixel size 6.5 microns) using $2 \times 2$ pixel binning (pixel size $216 \mathrm{~nm}$ ). For excitation of the fluorophores, three laser lines (DAPI: $405 \mathrm{~nm}$, Alexa488: $488 \mathrm{~nm}$, and Alexa647: $640 \mathrm{~nm}$ ), a fixed excitation dichroic mirror (405/488/561/640 nm), a fixed emission mirror (568 nm), and matched emission bandpass filters (DAPI: 445/45nm, Alexa488: 525/50 nm, and Alexa647: 676/29 nm) were used in separate exposures for each fluorophore. All wells were imaged using 9 randomly selected fields of view (FOVs). Each experiment included at least 3 technical replicates (wells on the same plate) per condition. Typically, approximately $1-2 \times 10^{3}$ cells were analyzed per well. Acquired images were processed using Columbus highcontent image analysis software (PerkinElmer, version 2.8.1 and 2.9.1). The analysis pipeline first identifies and segments nuclei using the DAPI image. Within identified and segmented nuclei, 53BP1 and $\square-\mathrm{H} 2 \mathrm{AX}$ foci were then detected using the Columbus spot-detection algorithm with low stringency detection parameters. A supervised machine learning algorithm (Fisher Linear Classifier) was used to classify 53BP1 and $\square$ $\mathrm{H} 2 \mathrm{AX}$ foci from background signals. The pre-trained model was fine-tuned by retraining to a dataset from each cell type to achieve robust and accurate classification of 53BP1 
and $\square-H 2 A X \square-H 2 A X$ foci. To reflect the distinct staining patterns of the two markers integrated spot intensity was measured for 53BP1 and mean nuclear intensity for $\square$ $\mathrm{H} 2 \mathrm{AX}$. The Columbus analysis sequence (.aas) files are available upon request.

Acquired images from the CV7000 were saved as .tif files and processed in Fiji, where only changes to adjust brightness and contrast on entire images were applied uniformly to all images of the same channel in a single figure panel.

\section{Statistical analysis}

Single-cell data was exported as tabular text files and further analyzed and plotted using R (R Core Team, 2019) and RStudio 1.3 (RStudio Team, 2020) using the tidyverse package ${ }^{41}$. All $p$-values for violin-boxplots of etoposide treated, untreated, and/or vehicle control samples were calculated using the Mann-Whitney-Wilcoxon test for pairwise comparisons, and the ANOVA test for multiple comparisons. Dot plots showing intra-assay variability between biological replicates in each condition also used MannWhitney-Wilcoxon and ANOVA to generate p-values. Unless indicated otherwise, experiments were performed at least as biological triplicates. The $R$ analysis scripts used to generate the plots in the figures are deposited at https://github.com/gallantk72/hti-ddr. 


\section{References}

1. Lindahl, T. \& Barnes, D. E. Repair of endogenous DNA damage. in Cold Spring Harbor Symposia on Quantitative Biology vol. 65 127-133 (Cold Spring Harbor Laboratory Press, 2000).

2. Jackson, S. P. \& Bartek, J. The DNA-damage response in human biology and disease. Nature 461, 1071-8 (2009).

3. Harper, J. W. \& Elledge, S. J. The DNA damage response: ten years after. Mol. Cell 28, 739-45 (2007).

4. Giglia-Mari, G., Zotter, A. \& Vermeulen, W. DNA damage response. Cold Spring Harb. Perspect. Biol. 3, a000745 (2011).

5. Reddy, P. T. et al. Production, Purification, and Characterization of $15 \mathrm{~N}$-Labeled DNA Repair Proteins as Internal Standards for Mass Spectrometric Measurements. in Methods in Enzymology vol. 566 305-332 (Academic Press Inc., 2016).

6. Chang, H. H. Y., Pannunzio, N. R., Adachi, N. \& Lieber, M. R. Non-homologous DNA end joining and alternative pathways to double-strand break repair. Nature Reviews Molecular Cell Biology vol. 18 495-506 (2017).

7. Paull, T. T. et al. A critical role for histone $\mathrm{H} 2 \mathrm{AX}$ in recruitment of repair factors to nuclear foci after DNA damage. Curr. Biol. 10, 886-895 (2000).

8. Collins, P. L. et al. DNA double-strand breaks induce H2Ax phosphorylation domains in a contactdependent manner. Nat. Commun. 11, 1-9 (2020).

9. Rogakou, E. P., Pilch, D. R., Orr, A. H., Ivanova, V. S. \& Bonner, W. M. DNA Double-stranded Breaks Induce Histone H2AX Phosphorylation on Serine 139. J. Biol. Chem. 273, 5858-5868 (1998).

10. Ward, I. M., Minn, K., van Deursen, J. \& Chen, J. p53 Binding Protein 53BP1 Is Required for DNA Damage Responses and Tumor Suppression in Mice. Mol. Cell. Biol. 23, 2556-2563 (2003).

11. Zimmermann, M. \& de Lange, T. 53BP1: pro choice in DNA repair. Trends Cell Biol. 24, 108-117 (2014).

12. Mah, L. J., El-Osta, A. \& Karagiannis, T. C. ph2AX: A sensitive molecular marker of DNA damage and repair. Leukemia vol. 24 679-686 (2010).

13. Kurashige, T., Shimamura, M. \& Nagayama, Y. Differences in quantification of DNA double-strand breaks assessed by 53BP1/ $\mathrm{H} 2 \mathrm{AX}$ focus formation assays and the comet assay in mammalian cells treated with irradiation and N-acetyl-L-cysteine. J. Radiat. Res. 57, 312-7 (2016).

14. Heylmann, D. \& Kaina, B. The $\mathrm{YH}$ 2AX DNA damage assay from a drop of blood. Sci. Reports 2016 61 6, 1-9 (2016).

15. Lengert, N. et al. AutoFoci, an automated high-throughput foci detection approach for analyzing low-dose DNA double-strand break repair. Sci. Rep. 8, 17282 (2018).

16. Schumann, S., Eberlein, U., Muhtadi, R., Lassmann, M. \& Scherthan, H. DNA damage in leukocytes after internal ex-vivo irradiation of blood with the $\alpha$-emitter Ra-223. Sci. Rep. 8, 2286 (2018).

17. Djuzenova, C. S. et al. A prospective study on histone $\mathrm{y}-\mathrm{H} 2 \mathrm{AX}$ and 53BP1 foci expression in rectal 
carcinoma patients: correlation with radiation therapy-induced outcome. BMC Cancer 15, 856 (2015).

18. Jansen, R. J. et al. Detection of DNA damage in peripheral blood mononuclear cells from pancreatic cancer patients. Mol. Carcinog. 54, 1220-1226 (2015).

19. Collin, G., Huna, A., Warnier, M., Flaman, J.-M. \& Bernard, D. Transcriptional repression of DNA repair genes is a hallmark and a cause of cellular senescence. Cell Death Dis. 9, 259 (2018).

20. Kubben, N. \& Misteli, T. Shared molecular and cellular mechanisms of premature ageing and ageing-associated diseases. Nat. Rev. Mol. Cell Biol. 18, 595-609 (2017).

21. Bektas, A., Schurman, S. H., Sen, R. \& Ferrucci, L. Aging, inflammation and the environment. Experimental Gerontology vol. 105 10-18 (2018).

22. Scaffidi, P. \& Misteli, T. Lamin A-Dependent Nuclear Defects in Human Aging. Science (80-. ). 312, 1059-1063 (2006).

23. Liu, B. et al. Genomic instability in laminopathy-based premature aging. Nat. Med. 11, 780-785 (2005).

24. Pritchard, C. C. et al. Inherited DNA-Repair Gene Mutations in Men with Metastatic Prostate Cancer. N. Engl. J. Med. 375, 443-453 (2016).

25. Lodato, M. A. et al. Aging and neurodegeneration are associated with increased mutations in single human neurons. Science (80-. ). 359, 555-559 (2018).

26. Smart, D. J. et al. Assessment of DNA double-strand breaks and gammaH2AX induced by the topoisomerase II poisons etoposide and mitoxantrone. Mutat. Res. 641, 43-7 (2008).

27. Trzeciak, A. R., Barnes, J. \& Evans, M. K. A modified alkaline comet assay for measuring DNA repair capacity in human populations. Radiat. Res. 169, 110-121 (2008).

28. Berwick, M. \& Vineis, P. Measuring DNA repair capacity: Small steps. Journal of the National Cancer Institute vol. 97 84-85 (2005).

29. Biran, A. et al. Quantitative identification of senescent cells in aging and disease. Aging Cell 16, 661-671 (2017).

30. Lee, Y., Wang, Q., Shuryak, I., Brenner, D. J. \& Turner, H. C. Development of a high-throughput Y- $^{-}$ H2AX assay based on imaging flow cytometry. Radiat. Oncol. 2019141 14, 1-10 (2019).

31. Sykora, P. et al. Next generation high throughput DNA damage detection platform for genotoxic compound screening. Sci. Reports 201881 8, 1-20 (2018).

32. Bäckström, A. et al. A Sample Preparation Protocol for High Throughput Immunofluorescence of Suspension Cells on an Adherent Surface. J. Histochem. Cytochem. 68, 473-489 (2020).

33. Rajaraman, R., Fox, R. A., Vethamany, V. G., Fernandez, L. A. \& MacSween, J. M. Adhesion and spreading behaviour of human peripheral blood mononuclear cells (PBMC) in vitro. Exp. Cell Res. 107, 179-190 (1977).

34. Pegoraro, G. \& Misteli, T. High-Throughput Imaging for the Discovery of Cellular Mechanisms of Disease. Trends Genet. 33, 604-615 (2017).

35. Dunne, A., Jowett, M. \& Rees, S. Use of primary human cells in high-throughput screens. Methods 
Mol. Biol. 565, 239-257 (2009).

36. Bondza, S. et al. Real-time Characterization of Antibody Binding to Receptors on Living Immune Cells. Front. Immunol. 8, 455 (2017).

37. Mirzoeva, O. K. \& Petrini, J. H. J. DNA Damage-Dependent Nuclear Dynamics of the Mre11 Complex. Mol. Cell. Biol. 21, 281 (2001).

38. Smith, J., Mun Tho, L., Xu, N. \& A. Gillespie, D. The ATM-Chk2 and ATR-Chk1 Pathways in DNA Damage Signaling and Cancer. Adv. Cancer Res. 108, 73-112 (2010).

39. Sanchez, Y. et al. Control of the DNA Damage Checkpoint by Chk1 and Rad53 Protein Kinases Through Distinct Mechanisms. Science (80-. ). 286, 1166-1171 (1999).

40. Tumasian, R. A. et al. Skeletal muscle transcriptome in healthy aging. Nat. Commun. 12, 1-16 (2021).

41. Wickham, H. et al. Welcome to the Tidyverse. J. Open Source Softw. 4, 1686 (2019).

\section{Acknowledgments}

Research in the Misteli lab and at the NCI HiTIF is supported by funding from the Intramural Research Program of the National Institutes of Health $(\mathrm{NIH})$, National Cancer Institute, and Center for Cancer Research (ZIAABC010389). KG is an NCI iCURE Scholar.

\section{Author Contributions}

KG: Conceptualization, Methodology, Software, Validation, Formal Analysis, Data curation, Data visualization, Investigation, Writing-original draft preparation, Writing review and editing

AB: Methodology, Resources, Writing- original draft preparation, Writing-review and editing

MK: Resources, Writing- original draft preparation, Writing- review and editing

AL: Resources, Writing - review and editing

LF: Conceptualization, Resources, Writing- original draft preparation, Writing- review and editing, Supervision 
bioRxiv preprint doi: https://doi. org/10.1101/2021.10.20.465132; this version posted October 20,2021 . The copyright holder for this preprint

(which was not certified by peer review) is the author/funder. This article is a US Government work. It is not subject to copyright under 17 USC 105 and is also made available for use under a CCO license.

GP: Conceptualization, Methodology, Software, Formal Analysis, Writing-original draft preparation, Writing- review and editing, Supervision

TM: Conceptualization, Methodology, Funding, Formal Analysis, Investigation Writingoriginal draft preparation, Writing - review and editing, Supervision 


\section{Figure Legends}

Fig. 1) High-throughput imaging of DNA damage in human immune cells. a) Schematic representation of the high-throughput imaging methodology to detect DNA damage in $\mathrm{CD}^{+} \mathrm{T}, \mathrm{CD} 8^{+} \mathrm{T}, \mathrm{B}$ cells and monocytes. The pipeline involves a modified immunofluorescence assay, imaging using an automated high-content screening system, image analysis for segmentation and fluorescence intensity cellular features extraction, and statistical analysis. b) Determination of seeding efficiency using DAPI staining $\sim 15$ hours after seeding. Additional substrates were added to poly-D-lysine plates; cells were seeded using the BlueWasher liquid handler unless indicated otherwise. Values represent at least 48 replicate wells per condition; box represents first and third quartiles. c) Jurkat, BJAB, and THP-1 cells were seeded at densities ranging from $2-10 \times 10^{4}$ in a 384 -well format. The total number of detected nuclei by DAPI is indicated. $\mathrm{N}=4$ wells per condition with at least 467 cells per condition.

Fig. 2) Detection of DNA damage in immune cell lines using HTI. a) Jurkat cells were either treated with DMSO or treated with $30 \mu \mathrm{M}$ etoposide (ETP) for $1.5 \mathrm{hrs}$ before being subjected to HTI. DNA damage sites are indicated by an increase in signal intensity and foci formation of 53BP1 and $\square-\mathrm{H} 2 \mathrm{AX}$ detected by specific antibodies. Scale bar: $8 \mu \mathrm{m}$. b) Distribution of 53BP1 sum spot intensity in DMSO- or ETP-treated cells. ${ }^{* * * *}: \mathrm{p}<0.0001$. c) Distribution of $\gamma-\mathrm{H} 2 \mathrm{AX}$ mean nuclear intensity in DMSO or ETP-treated immune cells. ${ }^{* * *}: p<0.0001$ using Mann-Whitney-Wilcoxon test. Number of analyzed cells ( $n$ ) indicated for each sample. The values are representative of at least 3 independent experiments. Boxes represent the first and third quartiles of the distribution, the line represents the median and the whiskers represent the highest and lowest data points within 1.5 times the interquartile range (IQR) above the upper quartile and below the lower quartile. d) Two-dimensional kernel density contour plots of the correlation between 53BP1 sum spot intensity and $\square-\mathrm{H} 2 \mathrm{AX}$ mean nuclear intensity for both ETP-treated (blue) and untreated (red) immune cell lines for the dataset shown in panels $B$ and $C$. $N=3$ wells per sample with at least 197 cells per well. 
Fig. 3) Detection of DNA damage in $\mathrm{CD4}^{+} \mathrm{T}, \mathrm{CD8}{ }^{+} \mathrm{T}$, B cells and monocytes using HTI. a) $\mathrm{CD}^{+} \mathrm{T}$ cells were either left untreated or treated with $30 \mu \mathrm{M}$ ETP for $1.5 \mathrm{hrs}$ before being subjected to HTI. DNA damage sites are indicated by formation of 53BP1 and $\gamma-\mathrm{H} 2 \mathrm{AX}$ foci detected by specific antibodies. Scale bar: $10 \mu \mathrm{m}$. b) Distribution of 53BP1 sum spot intensity in DMSO- or ETP-treated cells. ${ }^{* * *}: p<0.0001$. c) Distribution of $\gamma$-H2AX mean nuclear intensity in DMSO- or ETP-treated immune cells. ${ }^{* * * *}: p<0.0001$ using Mann-Whitney-Wilcoxon test. Number of analyzed cells $(n)$ indicated for each sample. The values are representative of at least 3 experiments. Boxes represent the first and third quartiles of the distribution. The line represents the median. The whiskers represent the highest and lowest data points within 1.5 times the interquartile range above the upper quartile and below the lower quartile. d) Twodimensional kernel density contour plots of the correlation between 53BP1 sum spot intensity and $\square-\mathrm{H} 2 \mathrm{AX}$ mean nuclear intensity for both ETP-treated (blue) and untreated (red) immune cell lines for the dataset shown in panels $B$ and $C . N=3$ wells per sample with at least 185 cells per well.

Fig. 4) Inter-assay variability. a) Distribution of 53BP1 sum spot intensity in untreated, DMSO-treated, and ETP-treated $\mathrm{CD}^{+} \mathrm{T}$ cells from the same donor. Each dot represents the mean value of a treatment replicate. Black line represents mean integrated 53BP1 spot intensity and $\square-\mathrm{H} 2 \mathrm{AX}$ mean nuclear intensity ${ }^{*}$ : $\mathrm{p}<0.05,{ }^{* *}$ : $\mathrm{p}<$ $0.01,{ }^{* * *}: \mathrm{p}<0.001$. ANOVA=0.99. b) Distribution of $\gamma-\mathrm{H} 2 \mathrm{AX}$ nuclear intensity in untreated, DMSO-treated, and ETP-treated $\mathrm{CD} 4^{+} \mathrm{T}$ cells from the same donor. Each dot represents the mean $\gamma$-H2AX nuclear intensity for each treatment replicate. Black line represents the mean $\square-\mathrm{H} 2 \mathrm{AX}$ nuclear intensity for all three replicates. ${ }^{*}: \mathrm{p}<0.05$, ns: $p>0.05$. ANOVA=0.93. Statistics calculated using Mann-Whitney-Wilcoxon test and oneway ANOVA. $\mathrm{N}=1776-14059$ cells per sample.

Fig. 5) Batch processing of human primary immune cells for analysis of 53BP1 spot intensity and $\gamma-\mathrm{H} 2 \mathrm{AX}$ nuclear intensity. $\mathrm{CD} 4^{+} \mathrm{T}, \mathrm{CD}^{+} \mathrm{T}$, and monocytes of 10 
different donors were processed simultaneously, left untreated or treated with $30 \mu \mathrm{M}$ ETP for $1.5 \mathrm{hrs}$ and imaged in high-throughput format. 53BP1 sum spot intensity vs. $\gamma$ H2AX mean nuclear intensity were blotted for each donor for a) $C D 4^{+} \mathrm{T}$ cells, b) $\mathrm{CD}^{+} \mathrm{T}$ cells $\mathrm{c}$ ) monocytes. $\mathrm{N}=663-5693$ cells per sample.

\section{Supplementary Figures}

Supplementary Fig. 1) Detection of 53BP1 spot intensity in immune cells. 53BP1 positive cells were characterized in each treatment group for a) immune cell lines (Jurkat, BJAB, and THP-1) and b) $C D 4^{+} T$ cells, CD8+T cells, monocytes, and B cells. The same samples as in figures 2 and 3 are used.

Supplementary Fig. 2) List of donors used in batch processing assay. a) For each listed donor in Figure 5, donors were identified by age and sex. 
Fig. 1

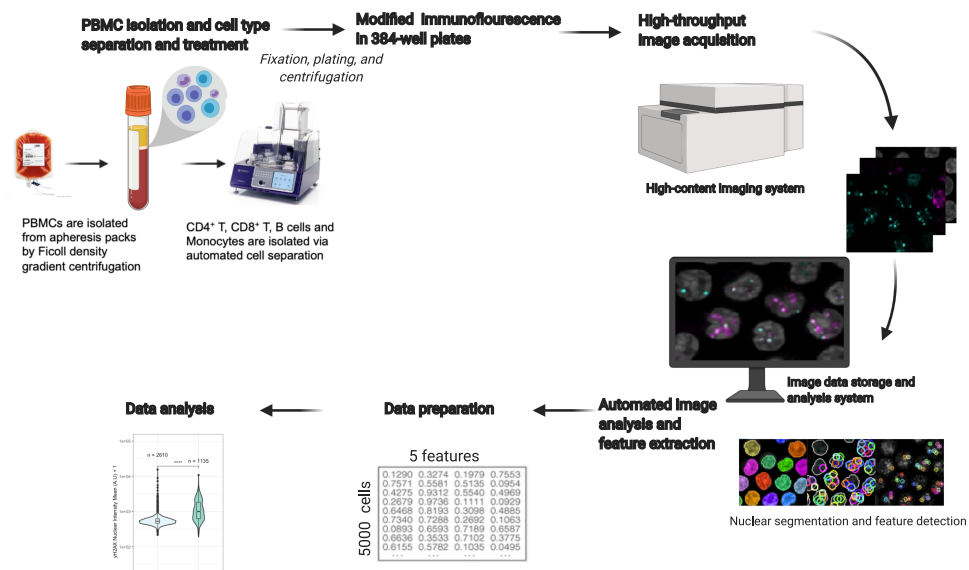

b
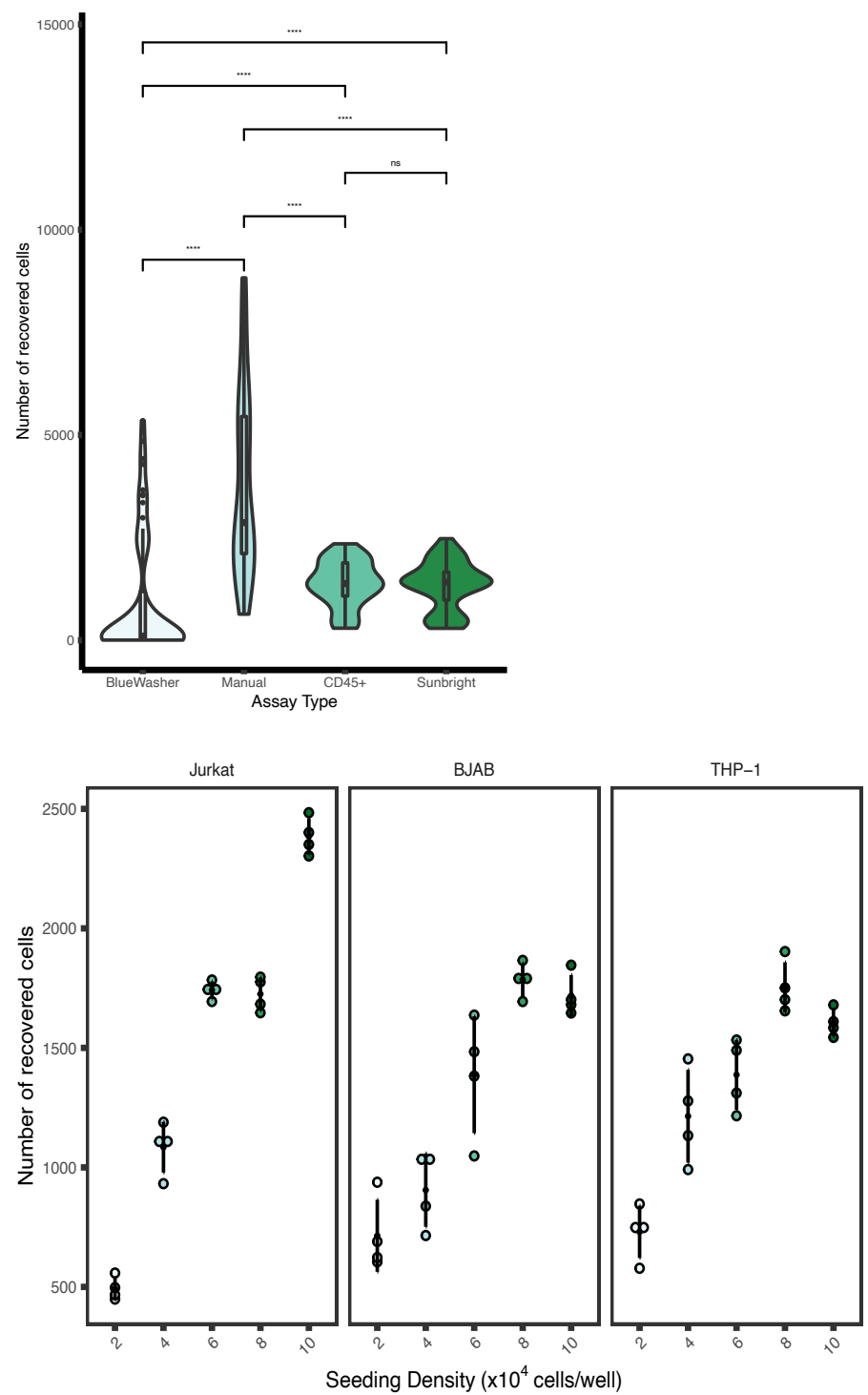
Fig. 2

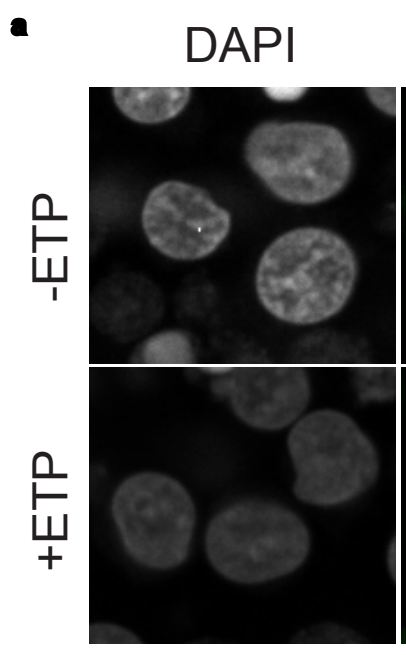

53BP1

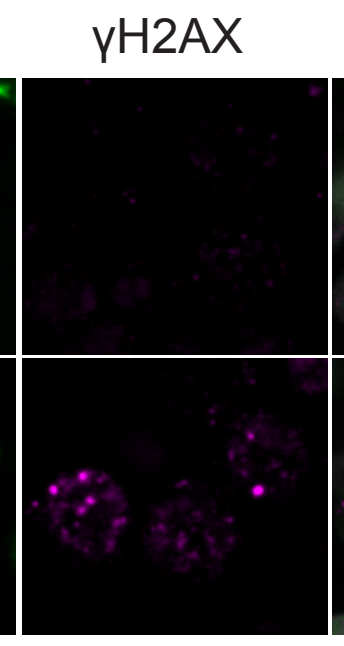

MERGE
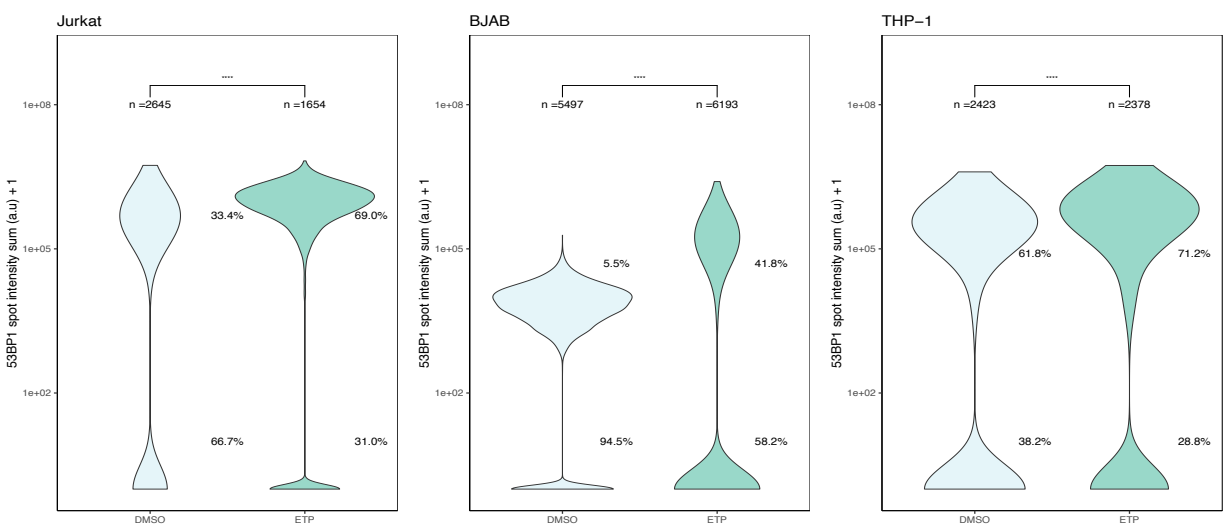

C
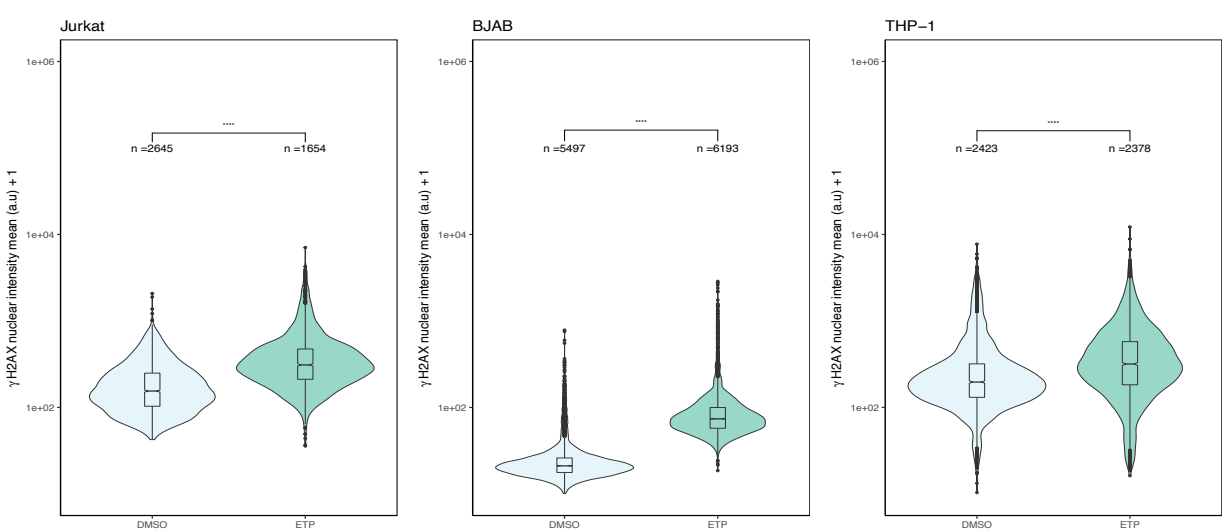

d
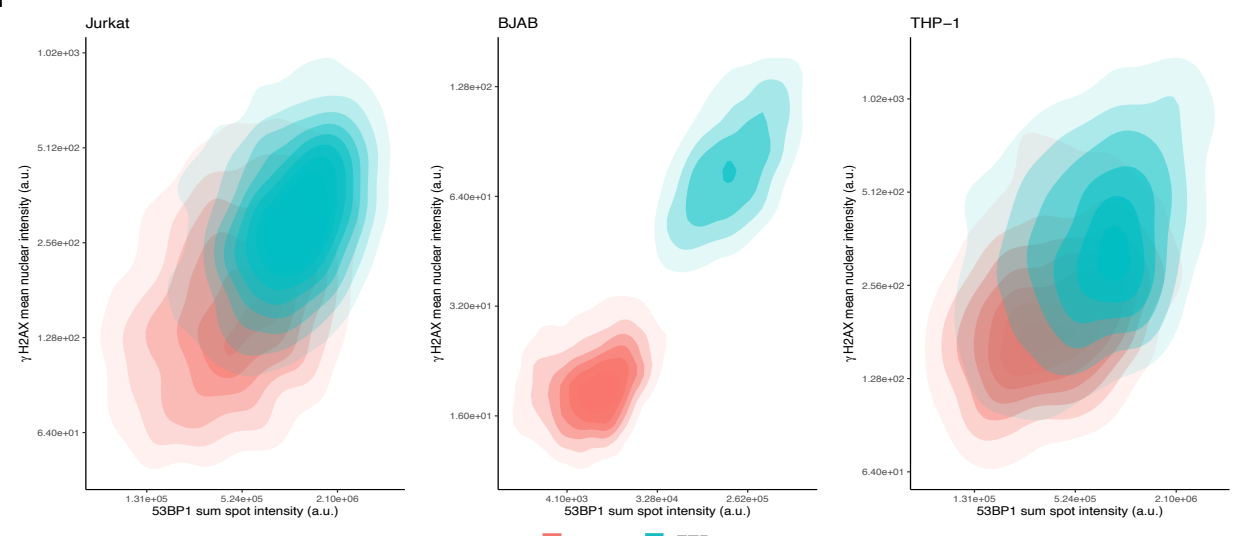


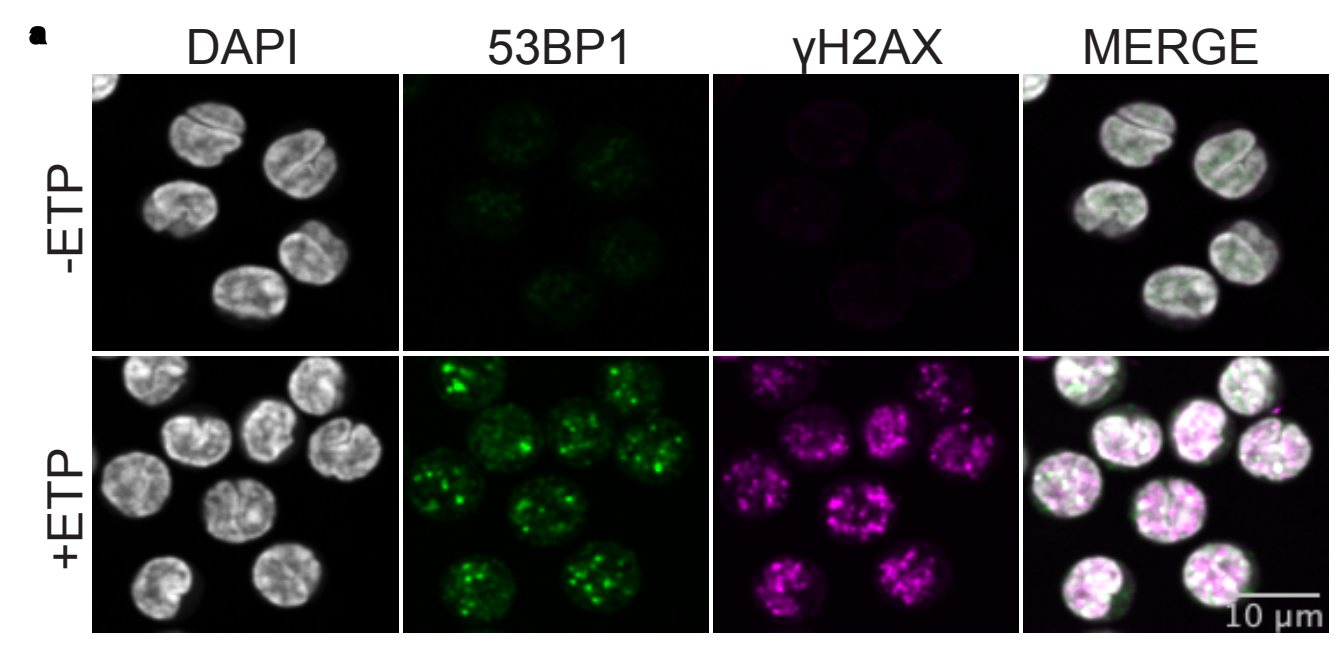

b

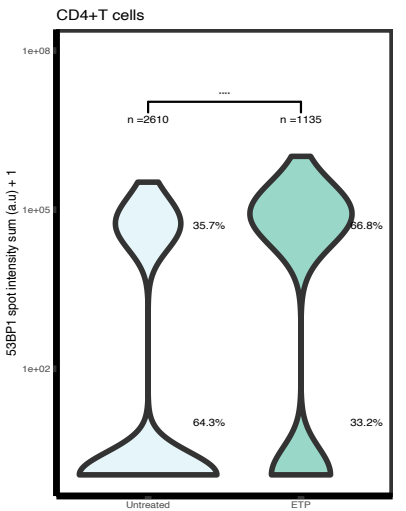

c
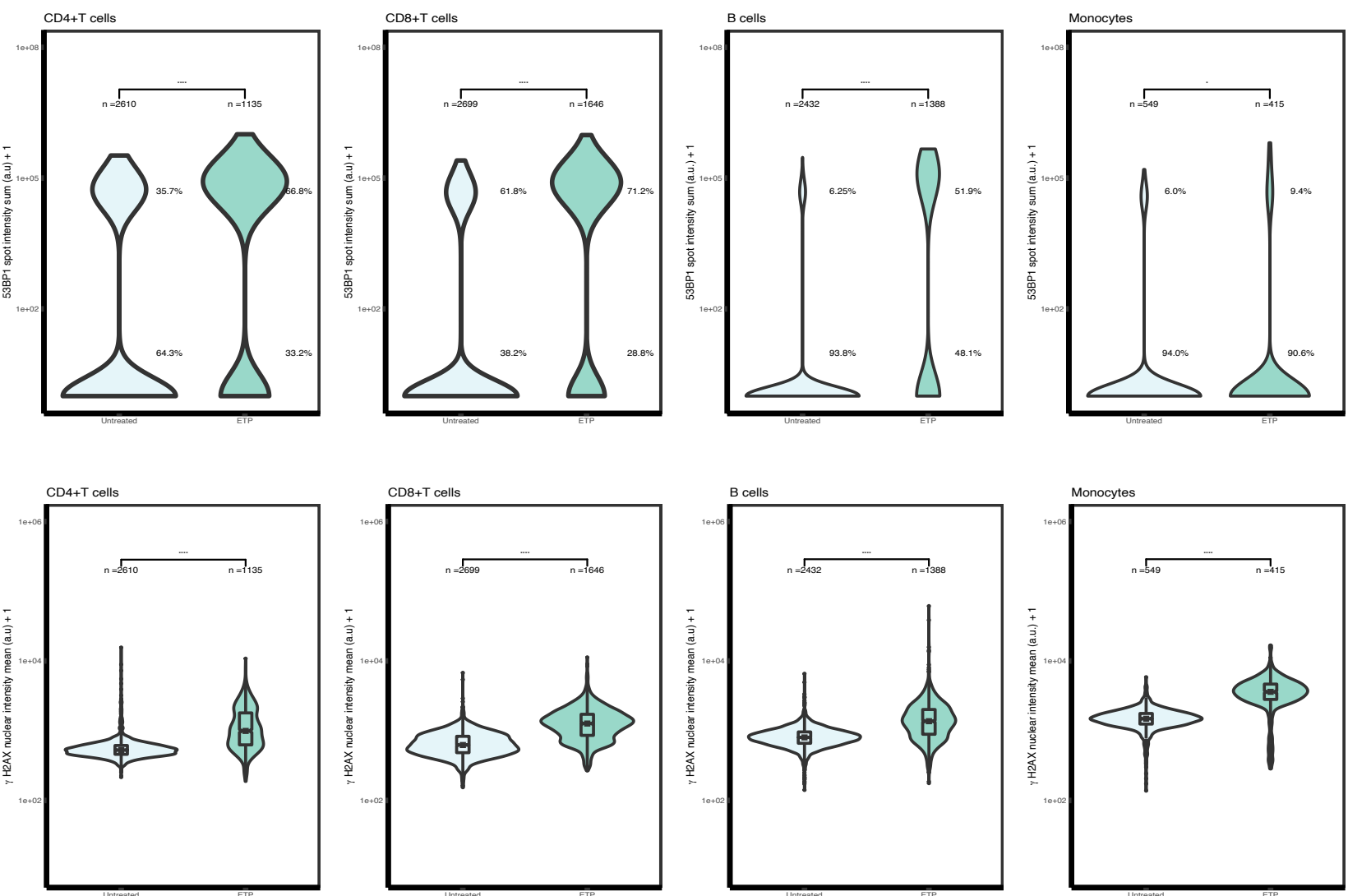

d
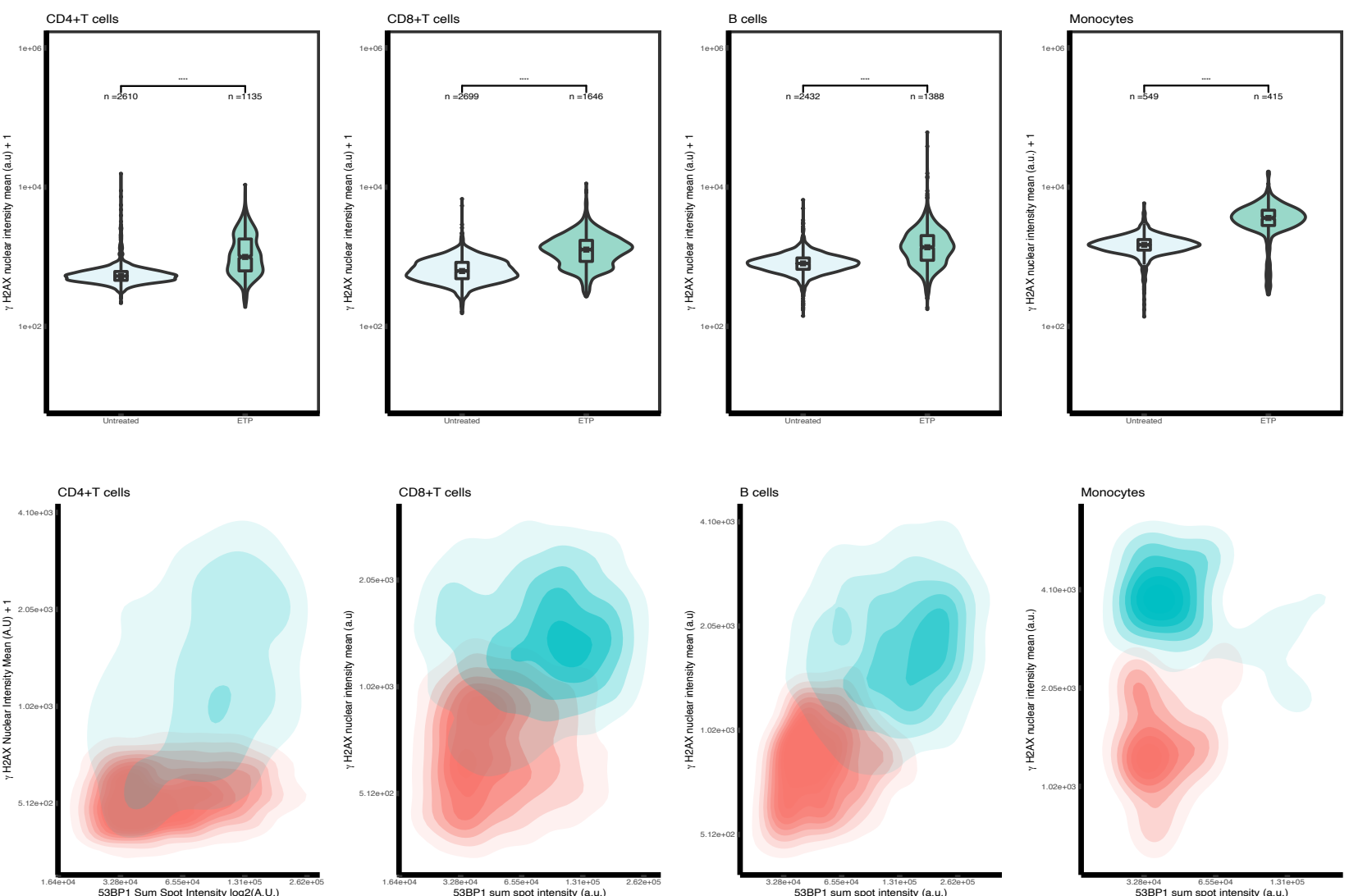

Fig. 3 


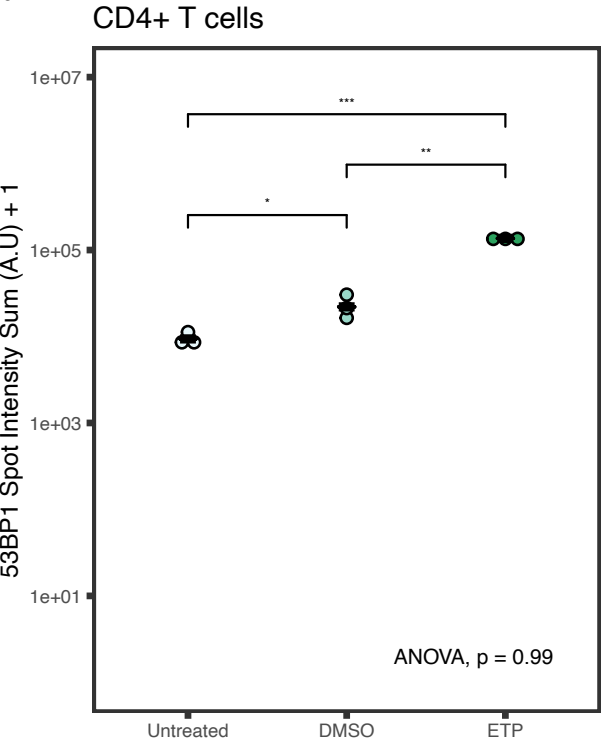

b

CD4+ T cells

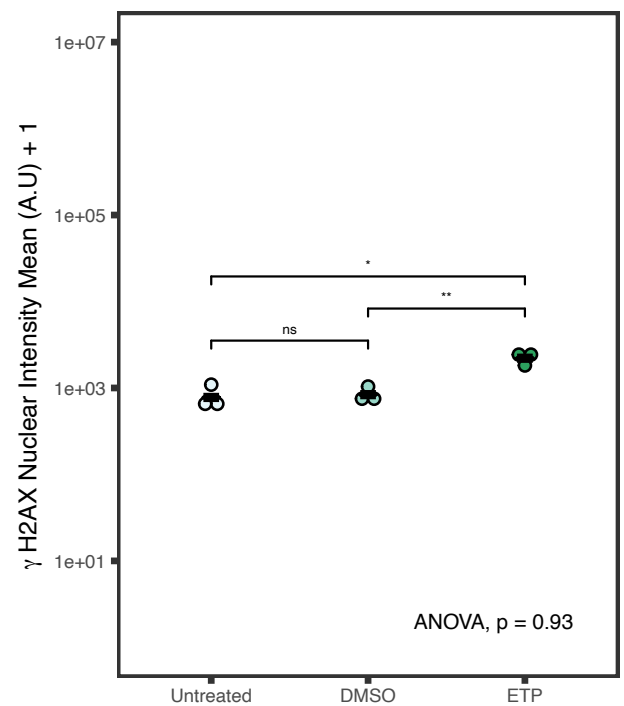

Fig. 4 
b

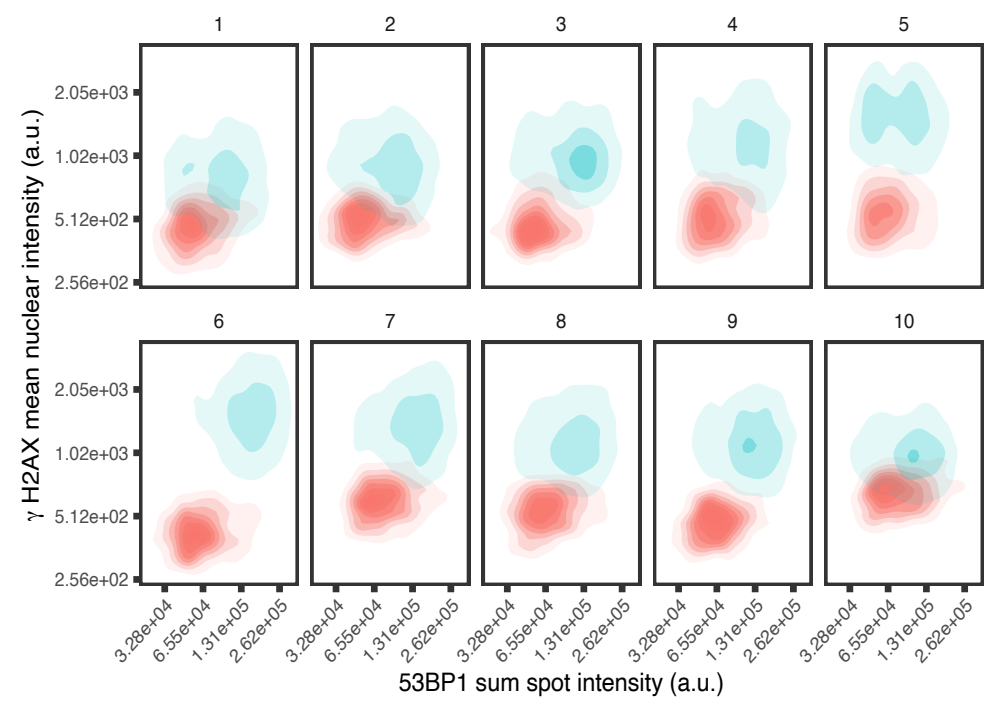

CD8+T cells

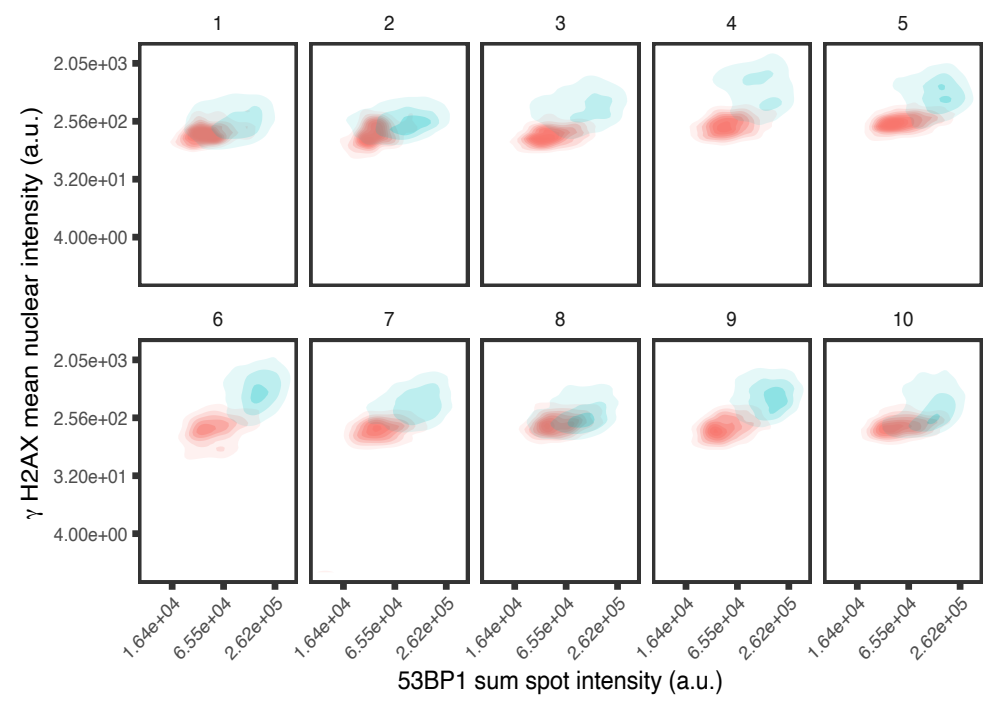

c

\section{Monocytes}

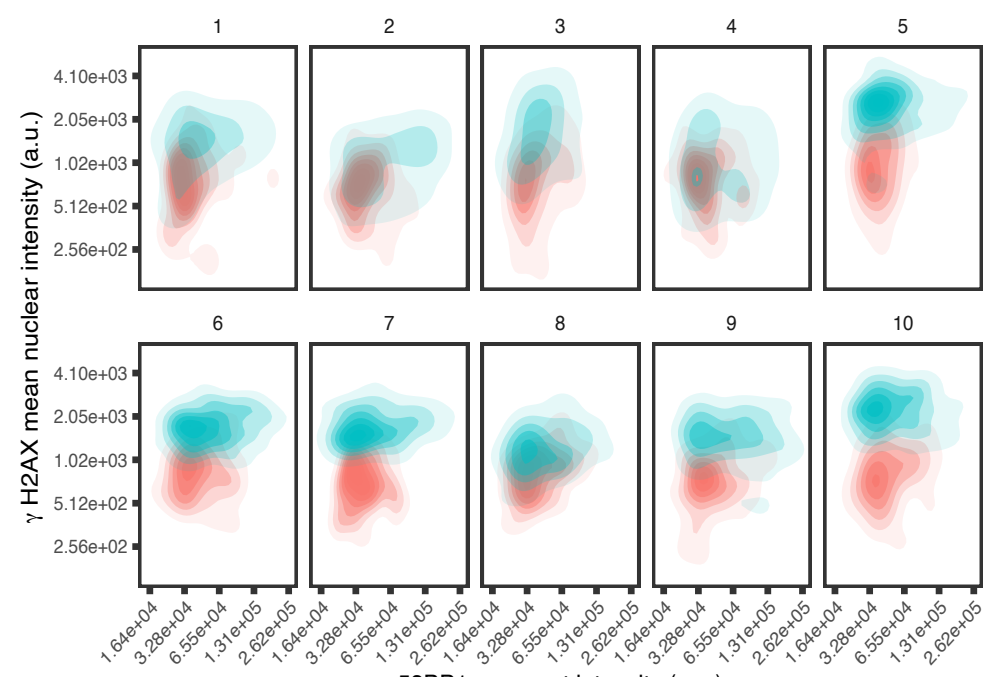

53BP1 sum spot intensity (a.u.) 